\begin{tabular}{|c|l|}
\hline Title & $\begin{array}{l}\text { A nomalous volumetric behavior of water-hex ane and water-decane mixtures in the vicinity of the critical region as } \\
\text { studied by infrared spectroscopy }\end{array}$ \\
\hline Author(s) & Ishikawa, Y uki; A rai, Sigenori; Furutaka, Seiya; Ikawa, Shun-ichi \\
\hline Citation & $\begin{array}{l}\text { The Journal of Chemical Physics, 122(204506), 1-5 } \\
\text { https://doi.org/10.1063/1.1906211 }\end{array}$ \\
\hline Issue Date & 2005-05-25 \\
\hline Doc URL & http://hdl.handle.net/2115/1393 \\
\hline Rights & Copyright $\odot 2005$ A merican Institute of Physics \\
\hline Type & article \\
\hline File Information & JCP122.pdf \\
\hline
\end{tabular}

Instructions for use 


\title{
Anomalous volumetric behavior of water-hexane and water-decane mixtures in the vicinity of the critical region as studied by infrared spectroscopy
}

\author{
Yuki Ishikawa, Sigenori Arai, Seiya Furutaka, and Shun-ichi Ikawa \\ Division of Chemistry, Graduate School of Science, Hokkaido University, \\ Sapporo 060-0810, Japan
}

(Received 17 January 2005; accepted 18 March 2005; published online 25 May 2005)

\begin{abstract}
Infrared spectra of binary mixtures of water with hexane and decane were measured at temperatures and pressures in the 473-648 $\mathrm{K}$ and 70-350 bar ranges, respectively. Volumetric concentrations of water and the hydrocarbons in the mixtures were obtained from absorption intensities of the fundamental $\mathrm{OH}$ stretching band of HDO and combination transitions of the hydrocarbons. Using both the concentrations, densities of the aqueous mixtures were estimated and compared with densities before mixing, which were calculated using literature densities of the neat liquids. It is found that anomalously large volume expansion on mixing occurs in the vicinity of the critical region of the mixtures. (C) 2005 American Institute of Physics. [DOI: 10.1063/1.1906211]
\end{abstract}

\section{INTRODUCTION}

When two different fluids are mixed, the total volume changes to a certain extent. The volume change, being called an excess volume of the mixture, is one of the most fundamental properties for characterizing the mixing state. So far a great number of experimental data on the excess volumes of various kinds of mixtures have been accumulated and used to understand the mixing states on the basis of intermolecular interaction. ${ }^{1}$ The relative change of the volume on the mixing at temperatures and pressures near ambient condition is usually small and typically a few percents. However, it becomes significantly large at some temperature-pressure conditions. Wormald and co-workers ${ }^{2-4}$ have studied binary mixtures of carbon dioxide with ethane, ethene, and sulfur hexafluoride and found that the excess volume in the nearcritical region is about two orders of magnitude larger than that for the liquid mixtures near ambient conditions. Xiao et $a l .{ }^{5}$ have reported that the excess molar volume of watermethanol mixtures shows significantly large sigmoidal negative-to-positive change at 0.44 of methanol molar fraction at $573.6 \mathrm{~K}$ and $137 \mathrm{bar}$, which was suggested to be a near-critical behavior. For dilute aqueous hydrocarbons, it has been reported that the partial molar volumes of the hydrocarbons become anomalously large near the critical point of pure water. ${ }^{6-8}$ These anomalous behaviors will be useful to gain an insight into relationship between macroscopic properties of the mixtures and the intermolecular interactions.

In the recent studies, we have found another type of intriguing volumetric behavior for binary mixtures of water with benzene, toluene, and ethylbenzene by in situ infrared and near-infrared measurements of high-temperaturepressure mixtures. ${ }^{9-11}$ On mixing of water with the hydrocarbons at certain temperature-pressure conditions, the total volumes expand anomalously. The relative volume change is particularly large at pressures a little above the vapor pressure of water and at temperatures above the critical end point of the three-phase equilibrium curve of the aqueous binary mixtures. For example, 7 times volume expansion has been found on mixing of water and benzene with a molar ratio of $0.82 / 0.18$ at $573 \mathrm{~K}$ and 100 bar. $^{11}$ Such an anomalously large volume expansion would be a characteristic of aqueous mixtures of hydrophobic hydrocarbons. Then it is intriguing whether aliphatic hydrocarbons, which are thought of more hydrophobic than the aromatics, exhibit the similar volumetric behavior. The present paper deals with in situ infrared measurements of water-hexane and water-decane mixtures at high temperatures and pressures. Densities of the mixtures were obtained from the component concentrations, which were estimated from infrared absorption intensities. As a reference, average densities before mixing were calculated using literature densities of neat liquids at the same temperatures and pressures. Comparing the densities before and after mixing, we have estimated relative volume changes on the mixing.

\section{EXPERIMENTAL METHOD AND RESULTS}

Spectroscopic measurements were performed using the same experimental setup and the high-pressure cell as described previously. ${ }^{12}$ Guaranteed reagent hexane and decane from Nacalai Tesque (Japan) and deuterium oxide (99.9\% D) from CEA (France) were used as received. Water specimens were mixtures of $\mathrm{H}_{2} \mathrm{O}$ and $\mathrm{D}_{2} \mathrm{O}$ with a molar ratio of $1: 10$ or 1:20. The ratios of the isotopic species, $\mathrm{H}_{2} \mathrm{O}, \mathrm{HDO}$, and $\mathrm{D}_{2} \mathrm{O}$, in the water specimens were estimated to be 1:20:100 or 1:40:400, and observed $\mathrm{OH}$ stretching absorption bands were assigned solely to HDO to a good approximation. Measurements of HDO in the mixtures have some advantages. First, it enables us to avoid the complication of spectra due to overlap of symmetric and antisymmetric stretching bands, and then makes band shape analysis simpler. In addition, it makes possible to keep the $\mathrm{OH}$ stretching absorption within a measurable range even at high temperatures, where solubility of water in hydrocarbons is considerably large and the ab- 

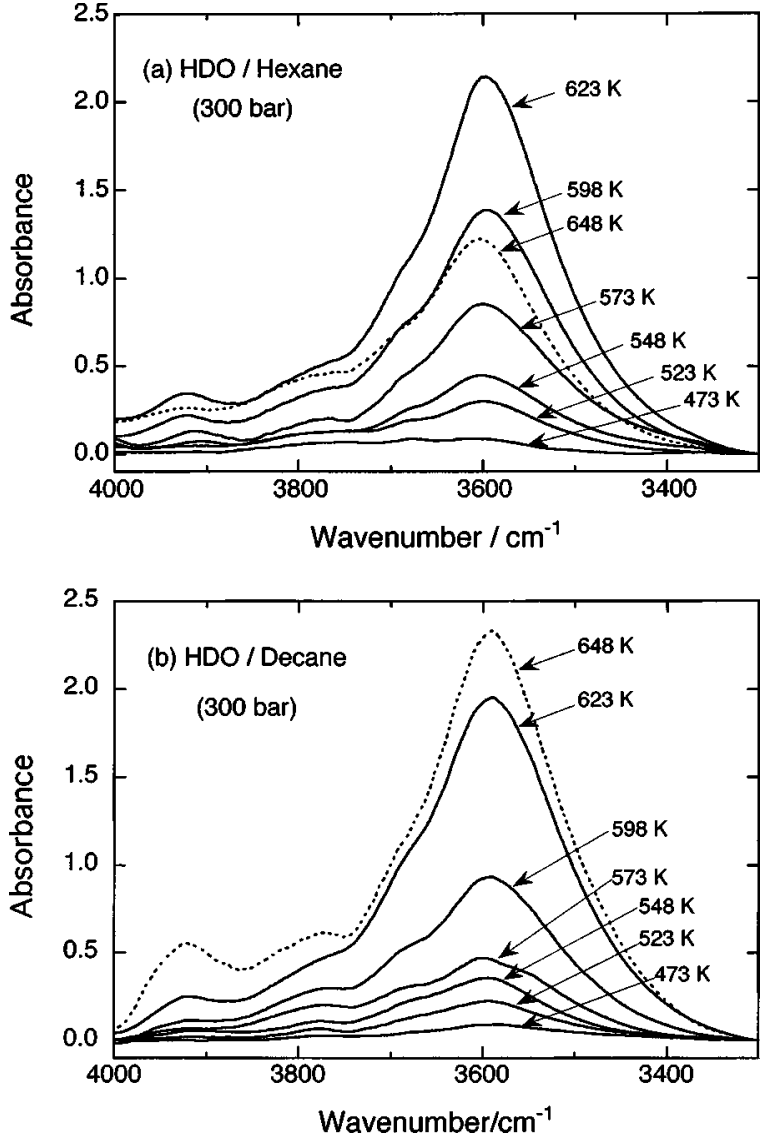

FIG. 1. Absorption spectra of HDO dissolved in hexane (a) and in decane (b) at various temperatures and constant pressure of 300 bar.

sorption would become too strong to measure when a neat $\mathrm{H}_{2} \mathrm{O}$ specimen is used. A small amount of the water specimen was put in the cell and then compressed liquid hydrocarbons were transmitted into the cell. The waterhydrocarbon interface was adjusted a little below the optical axis, and infrared absorption of the hydrocarbon-rich phase was observed with $2 \mathrm{~cm}^{-1}$ spectral resolution. At some of the experimental points of lower pressures and higher temperatures, however, observed spectra might be of the water-rich phase due to its anomalous volume expansion, as discussed later.

Figure 1 shows examples of observed absorption in the $\mathrm{OH}$ stretching region at various temperatures and at constant pressure of 300 bar, where spectra of neat hexane and decane observed at the same temperature and pressure were used as reference. Most part of the absorption is assigned to HDO, while a small absorption at about $3910 \mathrm{~cm}^{-1}$ is assigned to a combination transition of $\mathrm{D}_{2} \mathrm{O}$. The HDO band should consist of absorptions due to both hydrogen-bond-free and hydrogen-bonded $\mathrm{OH}$ groups. ${ }^{13}$ To obtain integrated intensity of the HDO absorption, we decomposed the absorption into several components with Gaussian and Lorentzian profiles and excluded contribution of the $\mathrm{D}_{2} \mathrm{O}$ band and uncertainty of the baseline which might be affected by possible incomplete cancellation of the solvent absorption. The absorption intensities increase remarkably with increasing temperature, indicating increase in water solubility in both the solvents. However, the intensity at $648 \mathrm{~K}$ reduces below that

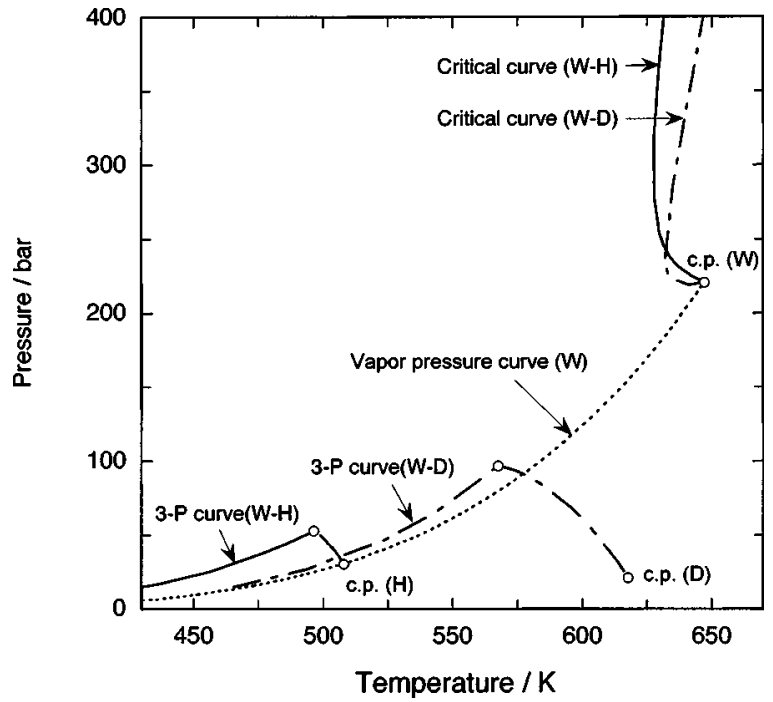

FIG. 2. A pressure-temperature phase diagram of water-hexane and waterdecane systems. $W, H$, and $D$ in parentheses stand for water, hexane, and decane, respectively. c.p., critical points (hexane, $507.9 \mathrm{~K}, 30.3$ bar: decane, $617.6 \mathrm{~K}, 21.0$ bar, water, $647.1 \mathrm{~K}, 220.6 \mathrm{bar}) .3-P$ curve: three-phase equilibrium curves (the end point for $W-H, 496.4 \mathrm{~K}, 52.8$ bar; the end point for $W-D, 567.6 \mathrm{~K}, 96.6$ bar).

at $598 \mathrm{~K}$ in hexane, whereas in decane the $648 \mathrm{~K}$ intensity becomes the largest in the present temperature range. This difference may be understood by difference in the position of the critical curve between the water-hexane and waterdecane systems as shown in Fig. 2, where the critical curves were drawn by use of literature data. ${ }^{14,15}$ The phase point of $648 \mathrm{~K}$ and $300 \mathrm{bar}$ is in the one-phase region in both the systems, but closer to the critical curve for water-decane than for water-hexane. Similar difference in temperature dependence of the absorption intensity has been found among water-aromatic hydrocarbon mixtures. ${ }^{12}$ However, it should be noted that the water concentration in the one phase region do not indicate the water solubility but depends on experimental condition.

The molar concentration of water in hydrocarbons can be estimated as $C_{w}=A_{\mathrm{OH}} / A^{m}$, where $A_{\mathrm{OH}}$ and $A^{m}$ denote the observed integrated intensity and the molar absorption intensity, respectively, of the $\mathrm{OH}$ stretching band of HDO. To estimate $A^{m}$, we have proposed an empirical expression by allowing for an internal-field effect and effect of hydrogenbond formation as follows:

$$
\begin{aligned}
& A^{m}=A_{0}^{m} / \Theta, \\
& \Theta=\frac{9 n}{\left(n^{2}+2\right)^{2}}, \\
& A_{0}^{m} /\left(10^{6} \mathrm{~cm} \mathrm{~mol}^{-1}\right)=3.0+5.37 \times 10^{-2}\left(3707-\nu_{0} / \mathrm{cm}^{-1}\right) \\
& +2.12 \times 10^{-4}\left(3707-\nu_{0} / \mathrm{cm}^{-1}\right)^{2},
\end{aligned}
$$

where, $\Theta$ denotes the internal-field correction factor ${ }^{16}$ given by the refractive index $n$ of the fluid mixture and $A_{0}^{m}$ is the molar absorption intensity as a function of the band center frequency $\nu_{0}$. Details of these expressions have been de- 

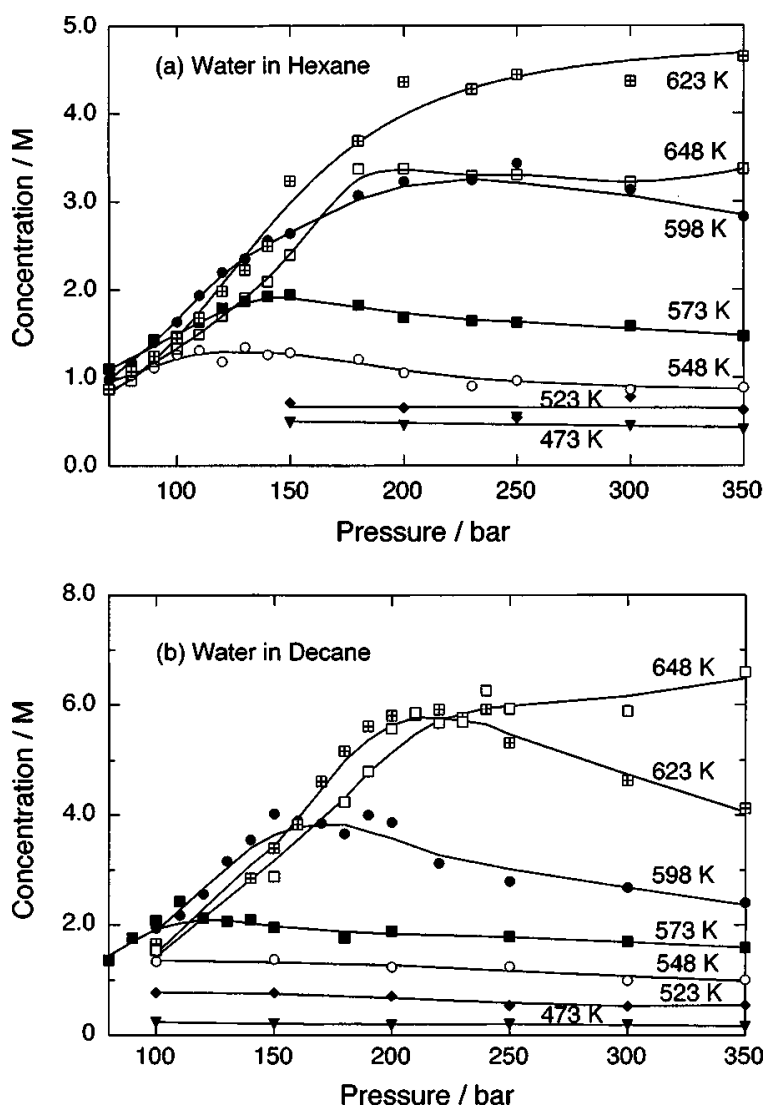

FIG. 3. Water concentrations in hexane and decane plotted against pressure at each temperature.

scribed previously. ${ }^{12}$ Briefly, the internal-field correction factor varies rather in the narrow range, $0.82-1.00$, and does not affect the similarity in the temperature-pressure dependence between the integrated intensity and the water concentration. Equation (3) has been derived as an empirical relationship between the integrated intensities and the peak frequencies, both of which vary significantly by hydrogen bonding. Since the observed bands are not symmetric and exhibit some structure, we have used the first moments of the absorption as the band center $\nu_{0}$. The resulting water concentrations are plotted in Fig. 3. The uncertainty of the values thus obtained may be in the range of $10 \%-20 \%$ (Ref. 12) except the results at the lowest temperature $473 \mathrm{~K}$, where the water solubility is very small and the results may have larger uncertainty.

To estimate concentrations of the hydrocarbons, we have used near-infrared absorption assigned to combination transitions of the $\mathrm{CH}$ stretching and bending vibrations. Examples of the observed spectra are shown in Fig. 4. It is found that the band profiles and peak positions of neat hydrocarbons and the water-hydrocarbon mixtures are very similar to each other. This fact suggests that the combination transitions are little affected by the environmental condition of the hydrocarbons, and we may assume that the molar absorption intensity is the same for the neat hydrocarbons and the aqueous mixtures. Integrated intensities of the neat hydrocarbons, $S_{\text {neat }}$, were obtained over the range of $4200-4500 \mathrm{~cm}^{-1}$ with a baseline leveled to a signal at $4500 \mathrm{~cm}^{-1}$ as shown in Fig. 4, and the molar absorption intensity is given by
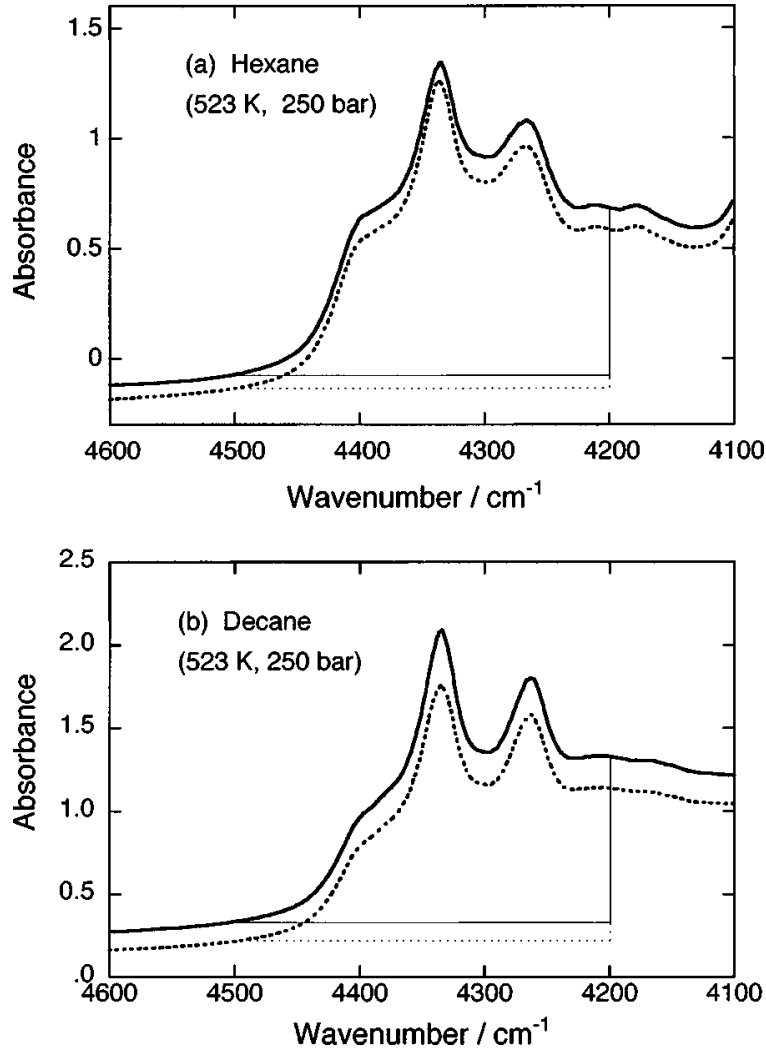

FIG. 4. Near-infrared absorption of hexane (a) and decane (b) in neat liquids (solid lines) and in aqueous mixtures (broken lines). Thin solid and dotted lines indicate base lines and integrated ranges.

$$
A_{\text {neat }}^{m}=S_{\text {neat }} /\left(C_{H}^{0} \cdot l\right),
$$

where $l$ is the sample thickness and $C_{H}^{0}$ denotes the molar concentration of neat hydrocarbons estimated from literature density data. ${ }^{17,18}$ The resulting molar absorption intensities were almost independent of temperature and pressure and we assume constant values: $(8.2 \pm 0.4) \times 10^{5} \mathrm{~cm} \mathrm{~mol}^{-1}$ for hexane and $(13.0 \pm 0.6) \times 10^{5} \mathrm{~cm} \mathrm{~mol}^{-1}$ for decane. This fact is consistent with the abovementioned assumption that the molar absorption intensity is independent of the molecular environment. The same situation has been observed for nearinfrared combination absorption of aromatic hydrocarbons. ${ }^{12}$

The molar concentrations of the hydrocarbons in the aqueous mixtures thus obtained were plotted in Fig. 5. It should be noted that the concentrations of hydrocarbons are very small and even smaller than the water concentrations in the region of high temperatures and low pressures. The spectra observed in that region may not be of the hydrocarbonrich phase but the water-rich phase which would significantly expand with dissolution of the hydrocarbons as discussed in the following section.

\section{DISCUSSION}

The volumetric behavior of the water-hydrocarbon mixtures are characterized by a relative volume change on the mixing by 

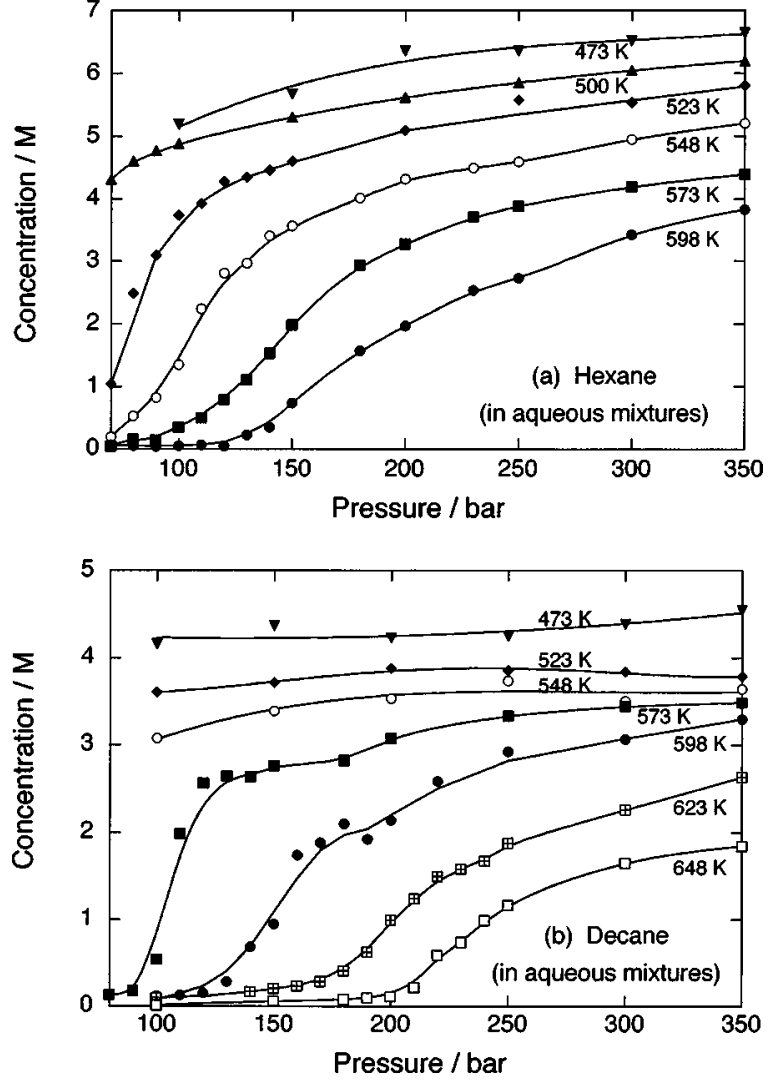

FIG. 5. Concentrations of hexane (a) and decane (b) in aqueous mixtures plotted against pressure at each temperature.

$$
\frac{\Delta_{\text {mix }} V}{V_{0}}=\frac{V_{\text {mix }}-V_{0}}{V_{0}}=\frac{\rho_{0}}{\rho_{\text {mix }}}-1,
$$

where

$$
\rho_{\text {mix }}=\left(C_{W} M_{W}+C_{H} M_{H}\right) / 1000
$$

denotes the density of the mixture given by the experimental concentrations, $C_{W}$ and $C_{H}$, and molar weights, $M_{W}$ and $M_{H}$, and

$$
\rho_{0}=\frac{C_{W} M_{W}+C_{H} M_{H}}{\left(C_{W} M_{W} / \rho_{W}\right)+\left(C_{H} M_{H} / \rho_{H}\right)}
$$

is the average density before mixing, $\rho_{W}$ and $\rho_{H}$ being densities of neat water and neat hydrocarbons taken from literatures. ${ }^{17,18}$ The resulting values of the relative volume change are plotted in Fig. 6. At temperatures below $523 \mathrm{~K}$ for the water-hexane system and $548 \mathrm{~K}$ for the water-decane system, the relative volume change is very small, being like that of an ordinary liquid mixture in the temperature-pressure region far from the critical condition. At higher temperatures, on the other hand, the relative volume change becomes anomalously large particularly at low pressures. For example, it reaches 13.2 for the water-hexane system at $573 \mathrm{~K}$ and 90 bar, which means 14.2 times volume expansion on the mixing. The pressures for the maximum volume expansion are 90, 130, and 170 bar at temperatures of 573, 598, and $623 \mathrm{~K}$, which are common to both the water-hexane and water-decane systems and are a little above the vapor pressures of neat water, 86, 120, and 165 bar, at the respective

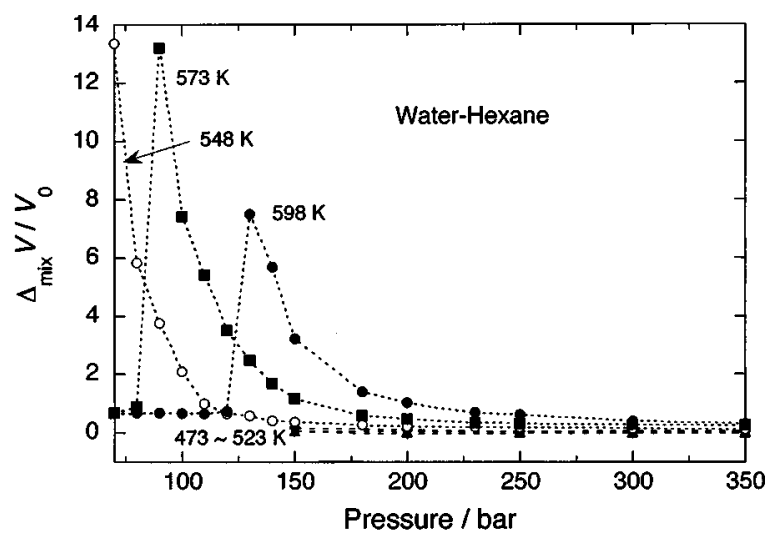

(a)

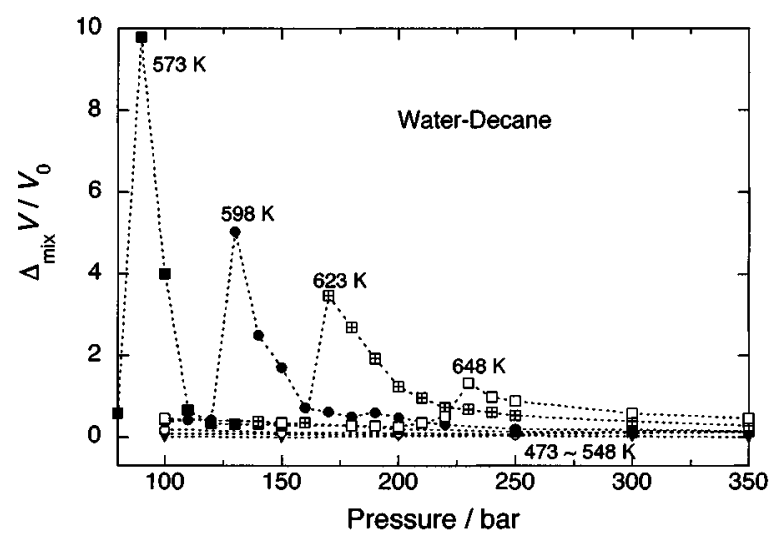

(b)

FIG. 6. Plots of the relative volume change on mixing for the water-hexane (a) and water-decane (b) systems against pressure at each temperature

temperatures. These results are consistent with the observation for the water-aromatic hydrocarbon systems, ${ }^{9-11}$ and suggest that the anomalous volume expansion on the mixing should be characteristic property of aqueous mixtures of hy-

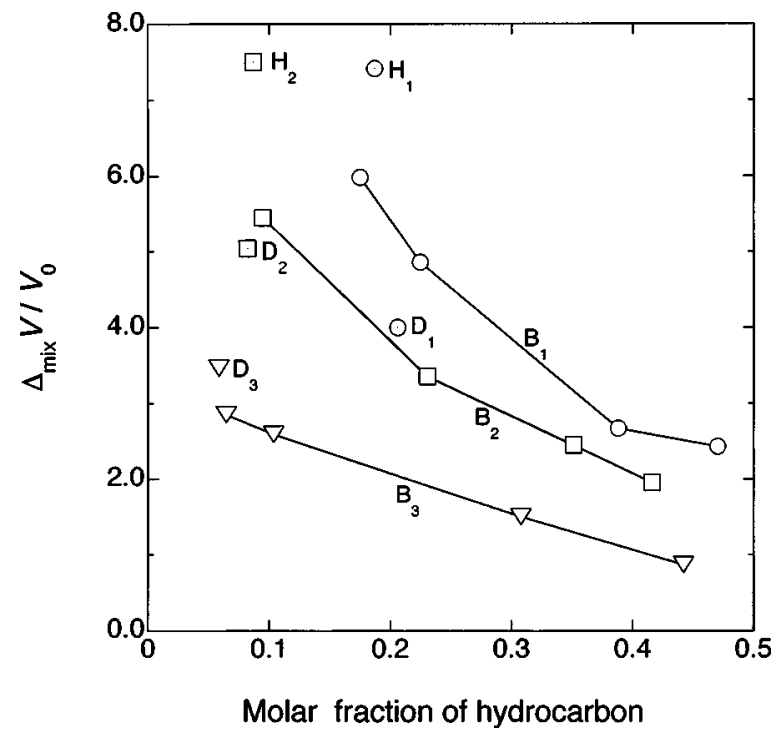

FIG. 7. Relative volume changes on mixing plotted against molar fraction of hydrocarbons for the water-benzene $\left[B_{1} \quad(573 \mathrm{~K}, 100 \mathrm{bar}) ; B_{2}\right.$ $\left.(598 \mathrm{~K}, 125 \mathrm{bar}) ; B_{3}(623 \mathrm{~K}, 175 \mathrm{bar})\right]$, water-hexane $\left[H_{1}(573 \mathrm{~K}, 100 \mathrm{bar})\right.$; $\left.H_{2} \quad(598 \mathrm{~K}, 130 \mathrm{bar})\right]$, and water-decane $\left[D_{1} \quad(573 \mathrm{~K}, 100 \mathrm{bar}) ; D_{2}\right.$ $\left.(598 \mathrm{~K}, 130 \mathrm{bar}) ; D_{3}(623 \mathrm{~K}, 170 \mathrm{bar})\right]$ systems. 
drophobic hydrocarbons. The detailed study on the waterbenzene system has suggested that the region where the anomalously large volume expansion occurs is a narrow region enclosed by an extended line of the three-phase equilibrium curve, the one-phase critical curve of the mixtures concerned and the vapor pressure curve of neat water [see Fig. 9 of Ref. 11].

Figure 7 shows plots of the relative volume changes for the water-hexane, water-decane, and water-benzene ${ }^{11}$ systems against molar fraction of hydrocarbons at a few temperature-pressure points. It proves that the relative volume changes for the three systems are not too much different from each other at the comparable conditions of temperature, pressure, and molar fraction. However, those for the waterhexane system are distinctly larger than for the water-decane and water-benzene systems. For the latter two systems, the relative volume expansions seem to be roughly the same allowing for a slight difference in pressure. Although, we could not examine dependence of the relative volume changes on the hydrocarbon fraction for the water-hexane and water-decane systems, it is probably similar to that for the water-benzene system shown in Fig. 7.

\section{ACKNOWLEDGMENT}

This work was supported by the Grant-in-Aid for Scientific Research from the Ministry of Education, Culture,
Sports, Science, and Technology of Japan (Grant No. 133440169).

${ }^{1}$ J. S. Rowlinson and F. L. Swinton, Liquids and Liquids Mixtures, 3rd ed. (Butterworth, London, 1982).

${ }^{2}$ C. J. Wormald, Thermochim. Acta 300, 169 (1997).

${ }^{3}$ C. J. Wormald and J. M. Eyears, Fluid Phase Equilib. 179, 139 (2001).

${ }^{4}$ C. J. Wormald and R. W. Hodgetts, Fluid Phase Equilib. 187-188, 391 (2001).

${ }^{5}$ C. Xiao, H. Bianchi, and P. R. Tremaine, J. Chem. Thermodyn. 29, 261 (1997).

${ }^{6}$ D. R. Bioggerstaff and R. H. Wood, J. Phys. Chem. 92, 1988 (1988).

${ }^{7}$ I. M. Abdulagatov, A. R. Bazaev, E. A. Bazaev, M. B. Saidakhmedova, and A. E. Ramazanova, Fluid Phase Equilib. 150-151, 537 (1998).

${ }^{8}$ I. M. Abdulagatov, A. R. Bazaev, E. A. Bazaev, M. B. Saidakhmedova, and A. E. Ramazanova, J. Chem. Eng. Data 43, 451 (1988).

${ }^{9}$ S. Furutaka and S. Ikawa, J. Chem. Phys. 117, 1682 (2002).

${ }^{10}$ S. Furutaka and S. Ikawa, Fluid Phase Equilib. 217, 181 (2004).

${ }^{11}$ Y. Jin and S. Ikawa, J. Chem. Phys. 121, 2694 (2004).

${ }^{12}$ S. Furutaka, H. Kondo, and S. Ikawa, Bull. Chem. Soc. Jpn. 74, 1775 (2001).

${ }^{13}$ S. Furutaka and S. Ikawa, J. Chem. Phys. 117, 751 (2002)

${ }^{14}$ Th. W. de Loos, W. G. Penders, and R. N. Lichtenthaler, J. Chem. Thermodyn. 14, 83 (1982).

${ }^{15}$ E. Brunner, J. Chem. Thermodyn. 22, 335 (1990).

${ }^{16}$ S. R. Polo and M. K. Wilson, J. Chem. Phys. 23, 2376 (1955).

${ }^{17}$ E. W. Lemmon, M. O. McLinden, and D. G. Friend, in NIST Chemistry WebBook, NIST Standard Reference Database Number 69, edited by P. J. Linstrom and W. G. Mallard (National Institute of Standards and Technology, Gaithersburg, MD, 2001) (http://webbook.nist.gov/).

${ }^{18}$ M. Gehrig and H. Lentz, J. Chem. Thermodyn. 15, 1159 (1983). 